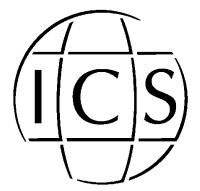

www.ics-elsevier.com

\title{
Cortical alpha rhythms in mild Alzheimer's disease. A multicentric EEG study
}

\author{
C. Babiloni ${ }^{\mathrm{a}, \mathrm{b}, *}$, G. Binetti ${ }^{\mathrm{c}}$, E. Cassetta $^{\mathrm{b}}$, D. Cerboneschi ${ }^{\mathrm{b}}$,

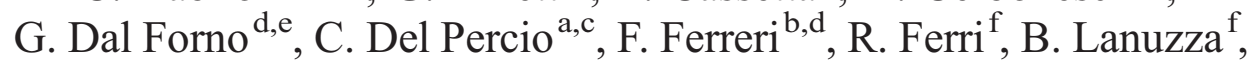 \\ C. Miniussi ${ }^{c}$, D.V. Moretti ${ }^{\mathrm{a}}$, F. Nobili ${ }^{\mathrm{g}}$, R.D. Pascual-Marqui ${ }^{\text {h, }}$, \\ G. Rodriguez ${ }^{\mathrm{g}}$, G.L. Romani ${ }^{\mathrm{i}}$, S. Salinari ${ }^{\mathrm{j}}$, F. Tecchio ${ }^{\mathrm{k}}$, P. Vitali ${ }^{\mathrm{f}}$, \\ O. Zanetti ${ }^{\mathrm{c}}$, F. Zappasodik ${ }^{\mathrm{k}}$, P.M. Rossini ${ }^{\mathrm{b}, \mathrm{c}, \mathrm{d}}$ \\ ${ }^{a}$ Dipartimento di Fisiologia Umana e Farmacologia, Università "La Sapienza", Rome, Italy \\ ${ }^{\mathrm{b}}$ A.Fa.R. Osp. FBF Isola Tiberina, Rome, Italy \\ ${ }^{\mathrm{c}}$ IRCCS "S. Giovanni di Dio FBF, Brescia, Italy \\ 'Università "Campus Biomedico", Rome, Italy \\ ${ }^{\mathrm{e}}$ Department of Neurology, Johns Hopkins University School of Medicine, Baltimore, USA \\ ${ }_{\mathrm{f}}^{\mathrm{f}}$ Department of Neurology, Oasi Institute for Research on Mental Retardation and Brain Aging, Troina, Italy \\ ${ }^{\mathrm{g}}$ Division of Clinical Neurophysiology (DIMI), University of Genova, Genova, Italy \\ ${ }^{\mathrm{h}}$ The KEY Institute for Brain-Mind Research, University Hospital of Psychiatry, Zurich, Switzerland \\ 'ITAB University Chieti and INFN, UdR L'Aquila, Italy \\ ${ }^{j}$ Dipartimento Informatica e Sistemistica Università "La Sapienza", Rome, Italy \\ ${ }^{\mathrm{k}}$ IFN-Consiglio Nazionale delle Ricerche (CNR) Unità MEG-Osp. FBF Isola Tiberina, Rome, Italy
}

\begin{abstract}
The study aimed at mapping (i) the distributed alpha $(8-13 \mathrm{~Hz})$ electroencephalography (EEG) sources specific for mild Alzheimer's disease (AD) compared with vascular dementia (VaD) in normal, elderly people (Nold) and (ii) the distributed alpha EEG sources sensitive to mild AD at different stages of severity. Resting EEG (10-20 electrode montage) was recorded from 48 mild $\mathrm{AD}, 20 \mathrm{VaD}$ and 38 Nold subjects. Both $\mathrm{AD}$ and $\mathrm{VaD}$ patients had 24 17 on their mini mental state examinations (MMSE). Alpha bands were subdivided in alpha 1 $(8-10.5 \mathrm{~Hz})$ and alpha $2(10.5-13 \mathrm{~Hz})$ subbands. Cortical alpha EEG sources were modeled by "low resolution brain electromagnetic tomography" (LORETA). Regarding issue (i), there was a decline of central, parietal, temporal and limbic alpha 1 sources specific to the mild AD group with respect to Nold and $\mathrm{VaD}$ groups. On the other hand, occipital alpha 1 sources showed a strong decline in mild $\mathrm{AD}$ compared with the $\mathrm{VaD}$ group. However, this finding was "unspecific" because a certain decline of these sources was also recognized in VaD compared with Nold. Regarding issue (ii), there was a lower power of occipital alpha 1 sources in the mild $\mathrm{AD}$ more severely diseased subgroup. On the whole, these findings stress the reliability of
\end{abstract}

* Corresponding author. Dipartimento di Fisiologia Umana e Farmacologia, Università "La Sapienza", P.le Aldo Moro 5, Rome 00185, Italy. Tel.: +39-6-49910989; fax: +39-6-49910917.

E-mail address: claudio.babiloni@uniroma1.it (C. Babiloni). 
modern technologies for EEG analysis as the LORETA approach to the study of cortical rhythmicity in resting mild AD. (C) 2004 Elsevier B.V. All rights reserved.

Keywords: Mild Alzheimer's disease (mild AD); Vascular dementia (VaD); Electroencephalography (EEG); Alpha rhythm; Low resolution brain electromagnetic tomography (LORETA)

\section{Introduction}

Electroencephalographic (EEG) rhythms are affected by Alzheimer's disease (AD) [1-3]. Compared with normal elderly subjects, Alzheimer's disease (AD) patients present an increase of delta (about $0.5-4 \mathrm{~Hz}$ ) and theta (about 4-8 Hz) mean power, along with a decrease of alpha (about $8-13 \mathrm{~Hz}$ ) and beta (about 13-30 Hz) mean power. EEG rhythms are also sensitive to the severity of dementia. Delta and/or theta rhythms do increase even in the earlier stages of AD [3] and seem to predict disease progression $[4,5]$. In normal subjects, the magnitude of alpha rhythm is maximal in scalp occipital areas. While alpha rhythm still peaks in the posterior scalp areas in mild AD patients, it is either equally distributed over the scalp or localizes more anteriorly with disease progression [5-7].

From a physiological point of view, EEG rhythms reflect the opening/closure ("gating function") of bidirectional connections among several cortical and subcortical (i.e., brain stem, thalamus) structures [8-10]. Therefore, a single dipole source indicates the "center of gravity" of the distributed cortical sources generating the EEG rhythms. An alternative approach for the modeling of these sources is called "low resolution brain electromagnetic tomography" (LORETA) [11,12], which uses thousands of dipole sources within a 3D brain model coregistered into Talairach space [13].

The present multicentric study was aimed at defining (i) the distributed alpha $(8-13$ $\mathrm{Hz}$ ) EEG sources specific for mild AD compared with Vascular dementia (VaD) or normal aging (Nold) and (ii) the distributed alpha EEG sources sensitive to mild AD progression. For these aims, resting EEG was recorded from a large group of mild $\mathrm{AD}, \mathrm{VaD}$ and normal elderly (Nold) subjects. Alpha bands were subdivided into alpha $1(8-10.5 \mathrm{~Hz})$ and alpha $2(10.5-13 \mathrm{~Hz})$ subbands. Cortical sources of alpha EEG rhythms were modeled by LORETA solutions in macrocortical regions.

\section{Materials and methods}

We recruited 48 mild $\mathrm{AD}$ patients, $20 \mathrm{VaD}$ patients and 38 Nold subjects. All patients had a Mini Mental State Evaluation (MMSE) [14] with results ranging from 24 to 17. The mild AD patients were further subdivided into mild AD "- " (MMSE 24-21, 23 subjects) and mild AD "+" (MMSE 20-17, 25 subjects) to address the issue of the increase of the severity of mild AD. Table 1 shows a report of the mean values of relevant personal and clinical parameters of mild $\mathrm{AD}, \mathrm{VaD}$ and Nold subjects.

Specialized, clinical units recorded EEG in resting subjects (eyes closed) whose vigilance was continuously controlled to avoid drowsiness. EEG data were recorded (0.3-70 Hz band pass) from 19 electrodes positioned, according to the international $10-$ 20 system (Fp1, Fp2, F7, F3, Fz, F4, F8, T3, C3, Cz, C4, T4, T5, P3, Pz, P4, T6, O1, O2). 
Table 1

Personal and neuropsychological data of interest of the Nold, AD, VaD subjects

\begin{tabular}{llll}
\hline & Nold & Mild AD & VaD \\
\hline$N$ & 38 & 48 & 20 \\
AGE (years) & $67.5( \pm 1.3$ S.E. $)$ & $73.7( \pm 1.3$ S.E. $)$ & $76.4( \pm 1.2$ S.E. $)$ \\
GENDER (F/M) & $19 / 19$ & $39 / 9$ & $10 / 10$ \\
MMSE & $29.2( \pm 0.2$ S.E. $)$ & $20.2( \pm 0.3$ S.E. $)$ & $20.4( \pm 1.1$ S.E. $)$ \\
EDUCATION (years $)$ & $8( \pm 0.7$ S.E. $)$ & $5.5( \pm 0.5$ S.E. $)$ & $9.7( \pm 1$ S.E. $)$ \\
\hline
\end{tabular}

A digital FFT-based power spectrum analysis (Welch technique, Hanning windowing function, no phase shift) computed the power density of alpha EEG rhythms with $0.5 \mathrm{~Hz}$ frequency resolution. The following alpha subbands were considered: alpha $1(8-10.5 \mathrm{~Hz})$ and alpha $2(10.5-13 \mathrm{~Hz})$.

We employed LORETA for the EEG source analysis, which has been extensively tested with simulation paradigms [11,12]. LORETA computed 3D linear solutions (LORETA solutions) for the EEG inverse problem within a three-shell spherical head model including scalp, skull and brain compartments. The brain compartment was restricted to the cortical gray matter/hippocampus. This compartment included 2394 voxels ( $7 \mathrm{~mm}$ resolution), each voxel containing an equivalent current dipole. LORETA solutions consisted of current voxel density values able to predict EEG spectral power density at scalp electrodes. To enhance the "topographical" results, a "spatial" normalization was obtained by normalizing the LORETA current density at each voxel for the LORETA power density averaged across all frequencies $(0.5-30 \mathrm{~Hz})$ /voxels of the brain volume. These normalized, relative current values were then log transformed. We collapsed LORETA solutions at the frontal, central, temporal, parietal, occipital and limbic regions of the brain model coded into Talairach space.

Regional, normalized LORETA solutions were compared by two ANOVA analyses, using relative current density values as the dependent variable and subjects' age and education as covariates. The first ANOVA design focused on distributed alpha EEG sources specific to mild AD. Its factors (levels) were Group (mild AD, VaD, Nold), Band (alpha 1, alpha 2) and ROI (central, frontal, parietal, occipital, temporal, limbic). The second ANOVA design focused on distributed alpha EEG sources sensitive to the severity of mild AD. Its factors (levels) were Group (Nold, mild AD - , mild AD+), Band (alpha 1, alpha 2) and ROI (central, frontal, parietal, occipital, temporal, limbic).

\section{Results}

Fig. 1 maps the grand average of LORETA solutions modeling distributed alpha EEG sources in Nold, mild AD (MMSE 24-17) and VaD groups. In the Nold group, alpha sources had strong magnitude and were distributed mainly in the parieto-occipital regions. Relative current density prevailed in alpha 1 compared with alpha 2 sources. Compared to the Nold group, the mild AD group showed a dramatic reduction of parieto-occipital alpha 1 sources. Compared to the $\mathrm{AD}$ group, the $\mathrm{VaD}$ group was characterized by a less dramatic decrease of parieto-occipital alpha 1 sources with respect to the Nold group.

Fig. 2 maps the grand average of LORETA solutions, modeling distributed alpha EEG sources in mild AD - (MMSE 24-21) and mild AD+ (MMSE 20-17) groups. Compared 


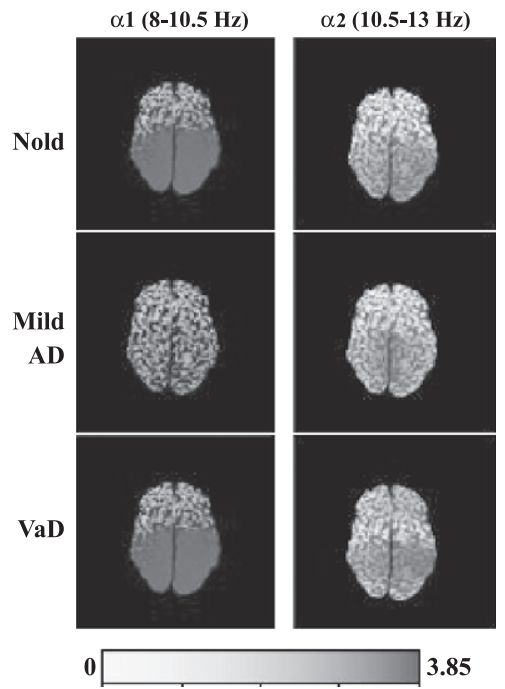

Fig. 1. Grand average of LORETA solutions (grey scale) modeling distributed alpha EEG sources in Nold, mild AD (MMSE 24-17) and VaD groups. The left side of the maps (top view) corresponds to the left hemisphere.

to the Nold group (see Fig. 1), occipital alpha 1 sources decreased in magnitude with the maximal severity of the disease (mild $\mathrm{AD}$ - to mild $\mathrm{AD}+$ ).

The ANOVA analysis, focusing on distributed alpha EEG sources specific to mild AD, showed a statistical ANOVA interaction $(p=0.03)$ among Group (mild AD, VaD, Nold), Band (alpha 1, alpha 2) and ROI (central, frontal, parietal, occipital, temporal, limbic) factors. Duncan post hoc showed a strong decline of central, parietal, temporal and limbic alpha 1 sources specific to mild AD with respect to Nold and VaD. Furthermore, occipital alpha 1 sources showed a strong decline in mild AD compared with VaD. However, this

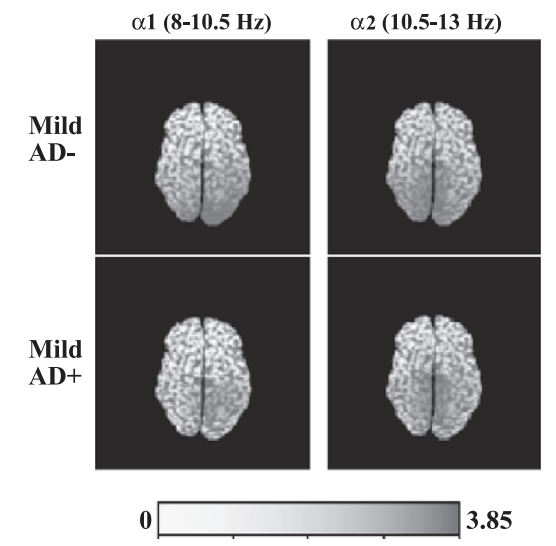

Fig. 2. Grand average of LORETA solutions (grey scale) modeling distributed alpha EEG in sources AD (MMSE 24-21) and mild AD+ (MMSE 20-17). The left side of the maps (top view) corresponds to the left hemisphere. 
finding was "unspecific" because a certain decline of these sources was also recognized in VaD compared with Nold.

The ANOVA analysis, focusing on distributed alpha EEG sources sensitive to the severity of mild AD, showed a statistical ANOVA interaction $(p=0.01)$ among Group (Nold, mild AD - , mild AD+), Band (alpha 1, alpha 2) and ROI (central, frontal, parietal, occipital, temporal, limbic) factors. Duncan post hoc indicated that the occipital alpha 1 sources had lower magnitude in mild AD - than Nold groups and lower magnitude in mild $\mathrm{AD}+$ than mild $\mathrm{AD}-$ groups.

\section{Discussion}

Cortical alpha 1 sources characterized mild $\mathrm{AD}$ from $\mathrm{VaD}$ and normal aging. Compared to $\mathrm{VaD}$ and normal aging, mild $\mathrm{AD}$ showed a significant decrease of alpha 1 sources in all cortical regions. In particular, the most specific marker for mild AD was the reduction in magnitude of central, parietal, temporal and limbic alpha 1 sources compared with normal aging and $\mathrm{VaD}$. Thus, it could be considered a marker specific for mild $\mathrm{AD}$. On the other hand, the reduction of the alpha 1 sources in the mild AD group respect to the control groups was clearly less evident in the central cortical region when compared with the parietal, occipital and temporal cortical regions. Furthermore, it was practically absent in the frontal region. The present results enlighten the so-called "anteriorization" of scalp alpha rhythms in $\mathrm{AD}$, repeatedly reported in previous studies using EEG mapping and single dipole localization $[5,6,14,15]$. Such an "anteriorization" may result from the fact that, in mild $\mathrm{AD}$, alpha 1 sources decline in magnitude much more in parieto-occipital than frontal cortical regions, thus producing a "virtual displacement" of the "center of gravity" of the alpha rhythm.

Compared to normal aging, magnitude reduction of widespread alpha 1 sources in mild AD can be explained in terms of an abnormal increase of cortical excitation or disinhibition during the resting state. This explanation is in line with previous evidence showing abnormal central EEG rhythms or evoked potentials in AD subjects who performed voluntary movements or received somatosensory stimuli [16,17].

In the present study, another important focus was on specific features characterizing distributed cortical sources of EEG rhythms during the different stages of severity of mild AD. Occipital alpha 1 sources had a stronger magnitude in Nold than mild AD - and in mild $\mathrm{AD}$ - than mild $\mathrm{AD}+$. These results, localized to the occipital cortical region, confirm previous scalp EEG evidence showing decreased alpha during AD progression $[1,2,7,15,18-20]$. The present abnormal sources of occipital EEG rhythms between mild $\mathrm{AD}$ at different stages of severity may be due to early pathological changes in extrastriate occipital areas [21] and their connections [22-24].

\section{References}

[1] C. Besthorn, et al., Discrimination of Alzheimer's disease and normal aging by EEG data, Electroencephalogr. Clin. Neurophysiol. 103 (2) (1997) 241-248.

[2] R. Chiaramonti, et al., Correlations of topographical EEG features with clinical severity in mild and moderate dementia of Alzheimer type, Neuropsychobiology 36 (3) (1997) 153-158.

[3] U. Schreiter-Gasser, T. Gasser, P. Ziegler, Quantitative EEG analysis in early onset Alzheimer's disease: 
correlations with severity, clinical characteristics, visual EEG and CCT, Electroencephalogr. Clin. Neurophysiol. 90 (4) (1994) 267-272.

[4] F. Nobili, et al., Timing of disease progression by quantitative EEG in Alzheimer's patients, J. Clin. Neurophysiol. 16 (6) (1999) 566-573.

[5] R. Ihl, et al., Topography of the maximun of the amplitude of EEG frequency in dementia of the Alzheimer type, Biol. Psychiatry 39 (1996) 319-325.

[6] R. Ihl, et al., Segmentation of the spontaneous EEG in dementia of the Alzheimer type, Neuropsychobiology 27 (4) (1993) 231-236.

[7] J.J. Claus, et al., The diagnostic value of electroencephalography in mild senile Alzheimer's disease, Clin. Neurophysiol. 110 (1999) 825-832.

[8] G. Pfurtscheller, C. Neuper, Event-related synchronization of mu rhythm in the EEG over the cortical hand area in man, Neurosci. Lett. 174 (1) (1994) 93-96.

[9] P. Nunez, Neocortical Dynamics and Human EEG Rhythms, Oxford Univ. Press, New York, 1995.

[10] G. Pfurtscheller, F.H. lopes da Silva, Event-related EEG/MEG synchronization and desynchronization: basic principles, Clin. Neurophysiol. 110 (11) (1999 Nov.) 1842-1857 (Review).

[11] R.D. Pascual-Marqui, C.M. Michel, LORETA (low resolution brain electromagnetic tomography): new authentic 3D functional images of the brain, ISBET Newsl. ISSN 5 (1994) 4-8.

[12] R.D. Pascual-Marqui, et al., Low resolution brain electromagnetic tomography (LORETA) functional imaging in acute, neuroleptic-naive, first-episode, productive schizophrenia, Psychiatry Res. 90 (3) (1999) $169-179$.

[13] J. Talairach, P. Tournoux, Co-planar Stereotaxic Atlas of the Human Brain, Thieme, Stuttgart, 1988.

[14] J.J. Claus, et al., Slowing on quantitative spectral EEG is a marker for rate of subsequent cognitive and functional decline in early Alzheimer disease, Alzheimer Dis. Assoc. Disord. 12 (3) (1998) 167-174.

[15] T. Dierks, et al., Spatial pattern of cerebral glucose metabolism (PET) correlates with localization of intracerebral EEG-generators in Alzheimer's disease, Clin. Neurophysiol. 111 (2000) 1817-1824.

[16] C. Babiloni, et al., Movement-related electroencephalographic reactivity in Alzheimer disease, NeuroImage 12 (2) (2000) $139-146$.

[17] R. Ferri, et al., Scalp topographic mapping of middle-latency somatosensory evoked potentials in normal aging and dementia, Neurophysiol. Clin. 26 (5) (1996) 311-319.

[18] F. Nobili, et al., Timing of disease progression by quantitative EEG in Alzheimer's patients, J. Clin. Neurophysiol. 16 (6) (1999) 566-573.

[19] G. Rodriguez, et al., EEG spectral profile to stage Alzheimer's disease, Clin. Neurophysiol. 110 (1999) $1831-1837$.

[20] C. Huang, et al., Discrimination of Alzheimer's disease and mild cognitive impairment by equivalent EEG sources: a cross-sectional and longitudinal study, Clin. Neurophysiol. 11 (2000) 1961-1967.

[21] R.A. Armstrong, et al., Neuropathological changes in the visual cortex in the Alzheimer's disease, Neurosci. Res. Commun. 6 (1990) 163-171.

[22] A. Cronin-Golomb, et al., Visual dysfunction in Alzheimer's disease: relation to normal aging, Ann. Neurol. 29 (1) (1991) 41-52.

[23] A. Cronin-Golomb, et al., Incomplete achromatopsia in Alzheimer's disease, Neurobiol. Aging 14 (5) (1993) 471-477.

[24] J.H. Morrison, P.R. Hof, C. Bouras, An anatomic substrate for visual disconnection in Alzheimer's disease, Ann. N. Y. Acad. Sci. 640 (1991) 36-43. 